Document downloaded from:

http://hdl.handle.net/10251/80091

This paper must be cited as:

Vidal-Ferràndiz, A.; González Pintor, S.; Ginestar Peiro, D.; Verdú Martín, GJ.; Asadzadeh, M.; Demazière, C. (2016). Use of discontinuity factors in high-order finite element methods. Annals of Nuclear Energy. 87:728-738. doi:10.1016/j.anucene.2015.06.021.

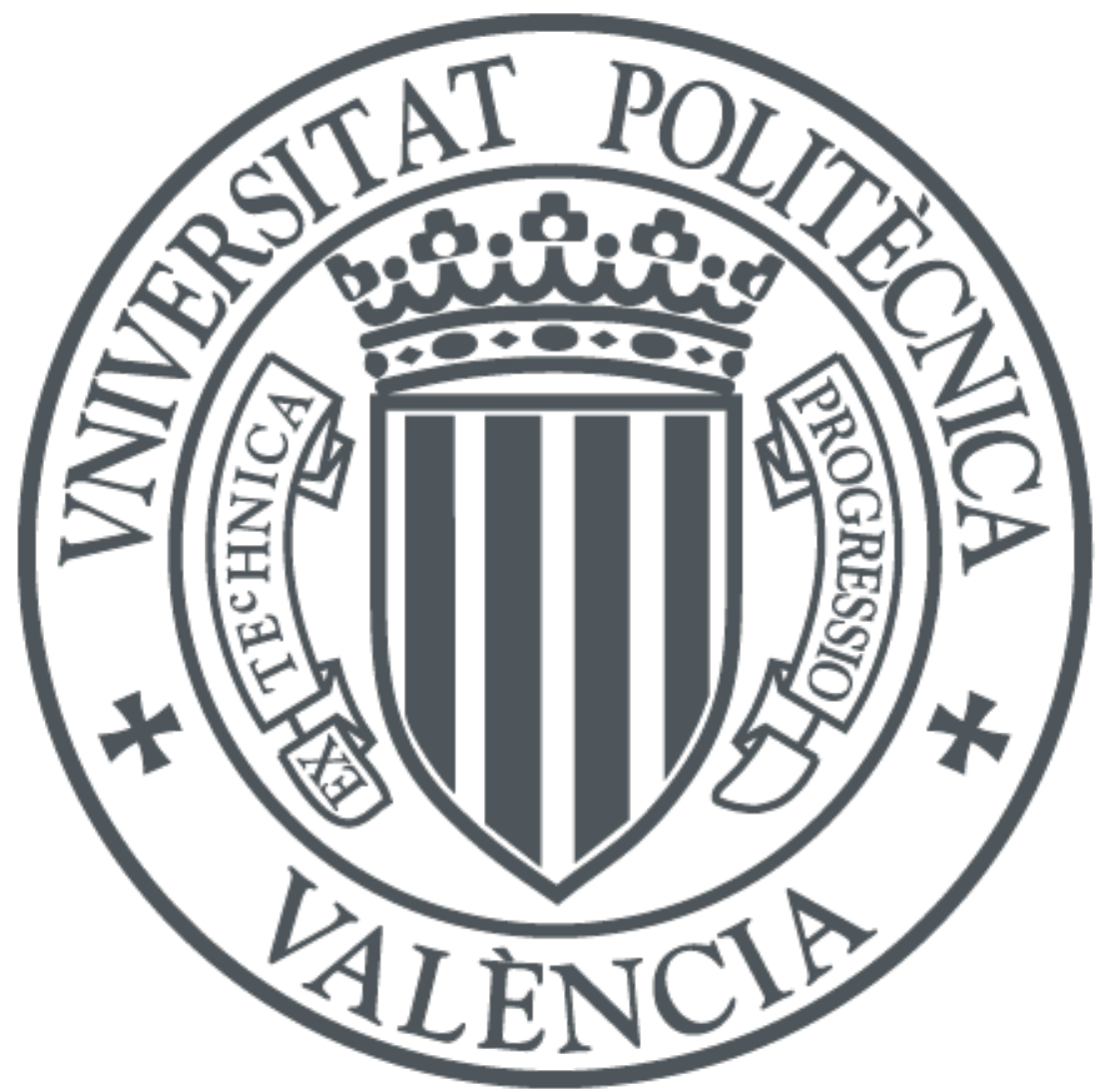

The final publication is available at

http://dx.doi.org/10.1016/j.anucene.2015.06.021

Copyright Elsevier Masson

Additional Information 


\title{
Use of discontinuity factors in high-order finite element methods
}

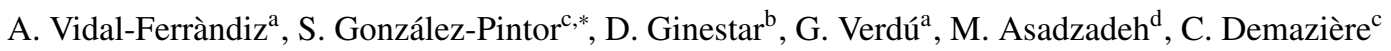 \\ ${ }^{a}$ Instituto de Seguridad Industrial: Radiofísica y Medioambiental, \\ Universitat Politècnica de València, \\ Camino de Vera s/n, 46022, València, Spain \\ ${ }^{b}$ Instituto Universitario de Matemática Multidisciplinar, \\ Universitat Politècnica de València, \\ Camino de Vera s/n, 46022, València, Spain \\ ${ }^{c}$ Department of Applied Physics, \\ Chalmers University of Technology, \\ SE-412 96, Göteborg, Sweden \\ ${ }^{d}$ Department of Mathematics, \\ Chalmers University of Technology and Göteborg University, \\ SE-412 96, Göteborg, Sweden
}

\begin{abstract}
The discontinuity factors are a technique widely used by nodal methods to minimize the error due to spatial homogenization of cross sections for a coarse mesh core calculation. In the present work, the introduction of discontinuity factors in a high-order finite element approximation of the neutron diffusion equation is investigated. More precisely, classical reference and assembly discontinuity factors are introduced in a discontinuous Galerkin finite element method stabilized using an interior penalty formulation for the neutron diffusion equation. The proposed method is tested solving different one and two-dimensional benchmark problems, showing that the discontinuity factors technique can be successfully introduced in the discontinuous Galerkin formulation.
\end{abstract}

Keywords: Discontinuity Factors, Finite Element Method, Neutron diffusion equation.

\section{Introduction}

Traditionally, the stationary neutron distribution inside a reactor core is approximated using the energy multigroup neutron diffusion equation (Stacey, 2007),

$$
-\vec{\nabla}\left(D_{g}(\vec{r}) \vec{\nabla} \phi_{g}(\vec{r})\right)+\Sigma_{a g}(\vec{r}) \phi_{g}(\vec{r})-\sum_{g^{\prime} \neq g}^{G} \Sigma_{s g^{\prime} g}(\vec{r}) \phi_{g^{\prime}}(\vec{r})=\frac{1}{k_{\mathrm{eff}}} \chi_{g} \sum_{g^{\prime}=1}^{G} v \Sigma_{f g^{\prime}}(\vec{r}) \phi_{g^{\prime}}(\vec{r}),
$$

where $\phi_{g}(\vec{r})$ is the neutron flux for the $g$-th energy group $(g=1, \ldots, G), D_{g}(\vec{r})$ the diffusion coefficient and $\Sigma_{a g}$, $\Sigma_{s g^{\prime} g}, v \Sigma_{f g^{\prime}}$, the nuclear cross sections, which are space dependent functions, and $k_{\text {eff }}$ is the multiplication factor of the reactor.

\footnotetext{
${ }^{*}$ Corresponding author

Email addresses: anvifer2@upv.es (A. Vidal-Ferràndiz), sebastian.gonzalez-pintor@chalmers.se (S. González-Pintor), dginesta@mat.upv.es (D. Ginestar), gverdu@iqn. upv.es (G. Verdú), mohammad@chalmers.se (M. Asadzadeh), demaz@chalmers.se (C. Demazière)
} 
To take into account a detailed description of the different cross sections associated with the different materials a reactor core is composed of is a very difficult task with the today's computer capabilities (Davidson et al., 2011), so a piece-wise homogenized version of the reactor core is used for the neutronic computations (Sanchez, 2009). Homogenization techniques are frequently applied in different problems of physics and engineering dealing with heterogeneous models. The basic idea of these methods is to substitute the heterogeneous components with homogeneous ones in such a way that the homogenized computation gives accurate average values. In this way, the homogenized parameters associated with heterogeneous regions have to be computed to preserve the average values that would be obtained from the heterogeneous solution.

This homogenization process is a source of error in the homogenized problem. To circumvent this homogenization error, while preserving region-wise homogenized parameters, flux discontinuity factors are a widely used technique (see section 2). Briefly, this technique forces jumps for the neutron flux between different homogenized regions and it can be easily implemented with nodal methods.

On the other hand, nodal methods may have some difficulties obtaining accurate solutions when large neutronic flux gradients appear (Palmtag, 1997). To obtain more accurate solutions, for example, high order finite element methods have been proposed (Hébert, 1985, 2008; Wang et al., 2009; Park and Cho, 2001; González-Pintor et al., 2009; VidalFerrandiz et al., 2014). Most of these finite element methods look for the neutronic flux as a continuous function in the reactor core. Thus, it is difficult to implement in such a methodology forced discontinuities between adjacent subdomains. Park and Cho (2001) proposed a high order finite element method that treats each one of the subdomains independently, where interface coupling conditions are imposed based on the incoming and outgoing partial currents along the subdomain interfaces. Here, a different approach is proposed using a high order discontinuous Galerkin finite element method where the jump condition for the neutron flux is imposed in a weak sense using interior penalty terms.

The remaining of the paper is organized as follows. In section 2, the homogenization process and the flux discontinuity factors are introduced. Then, in section 3, the formulation of the discontinuous Galerkin method for a homogenized reactor with assembly discontinuity factors is presented, both for one-dimensional and multi-dimensional geometries. Section 4 is devoted to present the method used to compute the reference discontinuity factors, both for one dimensional and multidimensional reactors. In section 5, the numerical results obtained with the proposed method are compared with a reference heterogeneous solution and with the results of a nodal method. Finally, the main conclusions of the paper are summarized in section 6.

\section{Homogenization and Flux Discontinuity Factors}

A first step in a homogenization methodology is to choose heterogeneous reactor properties that should be reproduced when the homogenized problem is solved. Usually, these quantities are the node averaged group reaction rates, the surface-averaged group currents and the $k$-effective of the reactor, which is implicitly conserved if the two aforementioned quantities are preserved. In this way, for every energy group $g$ and every nuclear cross section $\Sigma_{\alpha g}$, the equations (Smith, 1986),

$$
\int_{V_{k}} \hat{\Sigma}_{\alpha g}^{k} \hat{\phi}_{g}(\vec{r}) d \vec{r}=\int_{V_{k}} \Sigma_{\alpha g}(\vec{r}) \phi_{g}(\vec{r}) d \vec{r},
$$

and

$$
\int_{S_{k}}\left(\hat{D}_{g}^{k} \vec{\nabla} \hat{\phi}_{g}(\vec{r})\right) d \vec{S}=\int_{S_{k}}\left(D_{g}(\vec{r}) \vec{\nabla} \phi_{g}(\vec{r})\right) d \vec{S},
$$

must be satisfied, where $V_{k}$ and $S_{k}$ are the volume of the homogenized region $k$ and its limiting surface, respectively; $\phi_{g}$ is the solution of the heterogeneous problem and $\hat{\phi}_{g}$ is the solution obtained using the homogenized parameters $\hat{\Sigma}_{\alpha g}^{k}$ and $\hat{D}_{g}^{k}$.

The determination of the homogenized parameters from equation (2) requires the knowledge of the heterogeneous solution, $\phi_{g}$ and the homogenized solution $\hat{\phi}_{g}$, which depends on the homogenized parameters. To overcome this 
problem, an approximation must be done. The homogenized parameters are considered to be constant for every region $V_{k}$, thus, expression (2) can be rewritten as

$$
\hat{\Sigma}_{\alpha g}^{k}=\frac{\int_{V_{k}} \Sigma_{\alpha g}(\vec{r}) \phi_{g}(\vec{r}) d \vec{r}}{\int_{V_{k}} \phi_{g}(\vec{r}) d \vec{r}},
$$

where the average value of the homogeneous flux has been approximated by the average value of the heterogeneous flux, i.e.,

$$
\int_{V_{k}} \hat{\phi}_{g}(\vec{r}) d \vec{r} \approx \int_{V_{k}} \phi_{g}(\vec{r}) d \vec{r},
$$

and the diffusion coefficient can be expressed

$$
\hat{D}_{g}^{k}=\frac{\int_{S_{k}}\left(D_{g}(\vec{r}) \vec{\nabla} \phi_{g}(\vec{r})\right) d \vec{S}}{\int_{S_{k}} \vec{\nabla} \hat{\phi}_{g}(\vec{r}) d \vec{S}} .
$$

When looking at the homogenized diffusion coefficients in equation (6), we see that they are, in general, different for each surface, and this makes impossible to define constant diffusion coefficients in each homogenized region. To overcome this difficulty, some conditions imposed to the solution of the homogenized region have to be relaxed. Different approaches to relax these conditions lead to different homogenization methods. In the generalized equivalence theory (Smith, 1986), which is an extension of Koebke's homogenization method (Wagner and Koebke, 1983), flux discontinuity factors are introduced, relaxing the condition of continuity of the neutronic flux in the interior faces of the homogenized regions.

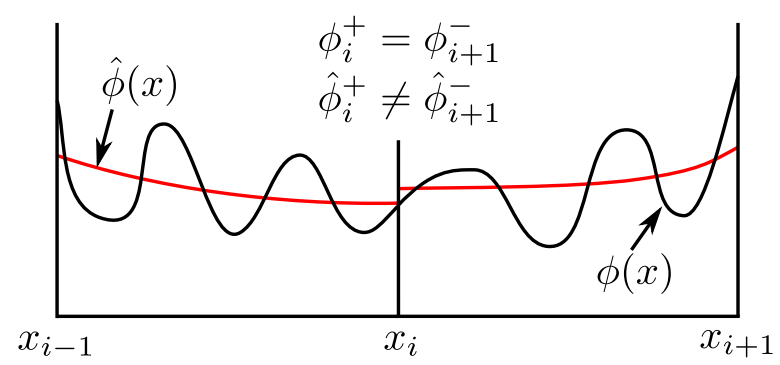

Figure 1: Reference discontinuity factors.

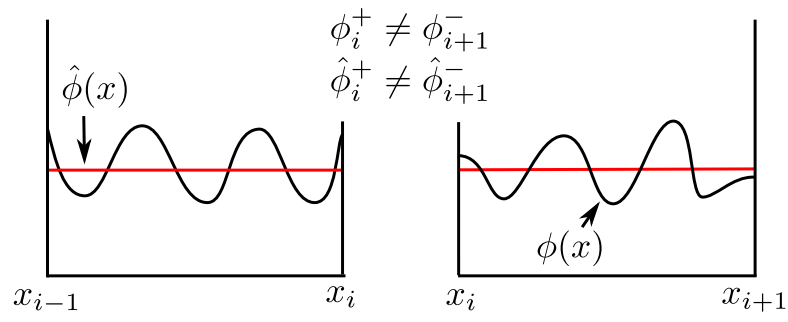

Figure 2: Assembly discontinuity factors. 
In this theory, the homogenized diffusion coefficients are computed as flux-volume weighted parameters, that is,

$$
\frac{1}{\hat{D}_{g}^{k}}=\frac{\int_{V_{k}} \frac{1}{D_{g}(\vec{r})} \phi_{g}(\vec{r}) d \vec{r}}{\int_{V_{k}} \phi_{g}(\vec{r}) d \vec{r}}
$$

where we have used the equation (5) to avoid the homogeneous flux in the formulation and, for a given interface $S_{j}$ limiting two adjacent homogenized regions, the energy-dependent discontinuity factors are defined as interface constants $f_{g, j}^{-}, f_{g, j}^{+}$, such that

$$
f_{g, j}^{-} \int_{S_{j}} \hat{\phi}_{g}^{-}(\vec{r}) d S=f_{g, j}^{+} \int_{S_{j}} \hat{\phi}_{g}^{+}(\vec{r}) d S,
$$

where $\hat{\phi}_{g}^{-}(\vec{r})$ and $\hat{\phi}_{g}^{+}(\vec{r})$ are the lateral (directional) limits of the homogenized flux in the surface $S_{j}$ viewed from the two different regions sharing this surface (see Figures 1 and 2). A possible definition of these discontinuity factors is

$$
f_{g, j}^{-}=\frac{\int_{S_{j}} \phi_{g}^{-} d S}{\int_{S_{j}} \hat{\phi}_{g}^{-} d S}, f_{g, j}^{+}=\frac{\int_{S_{j}} \phi_{g}^{+} d S}{\int_{S_{j}} \hat{\phi}_{g}^{+} d S},
$$

so continuity for the heterogeneous reconstructed flux is enforced. Condition (8) deals with averaged values in the surface $S_{j}$, so the generalized equivalence theory can be easily implemented into nodal methods, which use nodalaverage fluxes and surface-average currents (Smith, 1986).

At this point we need the value of the heterogeneous and homogeneous fluxes, $\phi_{g}(\vec{r})$ and $\hat{\phi}_{g}(\vec{r})$ respectively, to generate the discontinuity factors and the homogenized cross sections. Different choices for these fluxes generate different homogenization parameters. Two techniques are considered in this work. The first one consists of using the heterogeneous flux calculated for the whole reactor for generating the Reference Cross Sections (RXS), and appropriate fixed current boundary conditions from the heterogeneous solution, together with the global $k_{\text {eff }}$ and these RXS to generate the homogeneous flux in a particular region considered here as a fuel assembly. Thus, Reference Discontinuity Factors (RDF) are generated using equation (9) (See Figure 1). This technique provides very accurate homogenized parameters, but it requires the solution of the whole heterogeneous problem to generate the homogenized parameters, what makes it not practical. However, because of its reduced homogenization error, it is used here to verify that the discontinuity factors technique is successfully implemented within the interior penalty finite element method.

To circumvent the need of the whole core heterogeneous solution, the second technique studied here consists of considering that the heterogeneous flux is calculated for a single assembly with reflective boundary conditions. This approach would be accurate if the different assemblies composing the core were similar to the assembly being homogenized. Thus, with the assembly heterogeneous flux, we generate the Assembly homogenized Cross Sections (AXS), and the homogenized flux will be the solution of the homogenized problem with reflective boundary conditions, so we can define the Assembly Discontinuity Factors (ADF) with these fluxes (See Figure 2). This approach reduces the computational cost of obtaining a homogenized solution for the whole core, but introduces homogenization errors, which can be very large in a situation where the assemblies composing the core are very different to each other. The accuracy of the homogenized parameters can be increased by iterating between the solution of the homogenized problem and the homogenization process, using more accurate boundary conditions for the single assembly calculations that take into account the neighbourhood of the assembly. This kind of technique is not considered here, because the main focus of this work is the introduction of the flux discontinuity factors in the finite element formulation and not the homogenization process, that will be addressed in future works.

Moreover, different definitions of discontinuity factors exist that will not be considered here, such as the flux discontinuity ratios (Sanchez et al., 2013) or the current discontinuity factors (Sanchez, 2009). In this work only flux discontinuity factors are considered. 


\section{Discontinuous Galerkin method with interior penalty}

Discontinuous Galerkin methods, were introduced in the early seventies to study neutron transport equations (Reed and Hill, 1973; Lesaint and Raviart, 1974). They have become very popular to solve problems arising from hyperbolic type partial differential equations. Because of the versatility of these methods, they have been also applied to elliptic and parabolic problems (Arnold et al., 2000, 2002).

Discontinuous Galerkin (DG) methods define an approximation to the solution by means of a variational formulation that tries to enforce the partial differential equation of the model together with the boundary and continuity conditions for the solution in a weak sense (Brezzi et al., 2006). In this way, the DG method establishes a linear relationship between the residual of the approximation inside each element and its jumps across inter-element boundaries. This yields a formalism, that in a natural way, allows imposing jumps across the element interfaces as proposed in the generalized equivalence theory for homogenized reactors. It makes possible to use the finite element method for coarse-mesh reactor calculation after the homogenization procedure together with discontinuity factors, providing a suitable theoretical framework for developing new methodologies and more advanced schemes for the approximation of the homogenized neutron diffusion equation.

In the following, we present the Discontinuous Galerkin method proposed for the neutron diffusion equation with discontinuity factors, both for one and multi-dimensional problems.

\subsection{One-dimensional problems}

To show the method, we start with a source problem associated with the mono-energetic one-dimensional diffusion equation

$$
-\frac{\partial}{\partial x}\left(D \frac{\partial}{\partial x} \phi\right)+\Sigma_{a} \phi=q
$$

for a reactor $R$ discretized in $N+1$ cells with edges $x_{0}, x_{1}, \ldots, x_{N+1}$. Multiplying equation (10) by a test function, $v$, and integrating over $R$ we obtain

$$
-\int_{R} v \frac{\partial}{\partial x}\left(D \frac{\partial}{\partial x} \phi\right) d x+\int_{R} v \Sigma_{a} \phi d x=\int_{R} v q d x .
$$

Integrals defined over the whole domain in equation (11) can be written as the sum of integrals over each sub-domain as follows

$$
-\sum_{i=0}^{N} \int_{x_{i}}^{x_{i+1}} v \frac{\partial}{\partial x}\left(D \frac{\partial}{\partial x} \phi\right) d x+\sum_{i=0}^{N} \int_{x_{i}}^{x_{i+1}} v \Sigma_{a} \phi d x=\sum_{i=0}^{N} \int_{x_{i}}^{x_{i+1}} v q d x .
$$

After integrating by parts, equation (12) can be written as

$$
\sum_{i=0}^{N} \int_{x_{i}}^{x_{i+1}} \frac{\partial v}{\partial x} D \frac{\partial}{\partial x} \phi d x-\sum_{i=0}^{N} \int_{x_{i}}^{x_{i+1}} \frac{\partial}{\partial x}\left(v D \frac{\partial}{\partial x} \phi\right) d x+\sum_{i=0}^{N} \int_{x_{i}}^{x_{i+1}} v \Sigma_{a} \phi d x=\sum_{i=0}^{N} \int_{x_{i}}^{x_{i+1}} v q d x
$$

Assuming that the neutronic flux and the diffusion coefficients can be discontinuous and making use of lateral limits, expression (13) is expressed as

$$
-\sum_{i=0}^{N}\left(v_{i+1}^{-} D_{i+1}^{-} \frac{\partial}{\partial x} \phi_{i}^{-}-v_{i}^{+} D_{i}^{+} \frac{\partial}{\partial x} \phi_{i}^{+}\right)+D_{i} \sum_{i=0}^{N} \int_{x_{i}}^{x_{i+1}} \frac{\partial v}{\partial x} \frac{\partial \phi}{\partial x} d x+\sum_{i=0}^{N} \int_{x_{i}}^{x_{i+1}} v \Sigma_{a} \phi d x=\sum_{i=0}^{N} \int_{x_{i}}^{x_{i+1}} v q d x .
$$

where the piece-wise constant diffusion coefficient $D_{i}$ has moved outside of the integral, and $\phi_{i}^{ \pm}$is defined as the lateral limit of the flux at point $x_{i}$ (considering the flux as belonging to the previous or posterior subdomain), i.e.,

$$
\phi_{i}^{ \pm}=\lim _{x \rightarrow x_{i}^{ \pm}} \phi(x) .
$$


The first term of this equation, may be written as

$$
\begin{aligned}
T(\phi) & =\sum_{i=0}^{N}\left(v_{i}^{+} D_{i}^{+} \frac{\partial}{\partial x} \phi_{i}^{+}-v_{i+1}^{-} D_{i+1}^{-} \frac{\partial}{\partial x} \phi_{i}^{-}\right) \\
& =v_{0}^{+} D_{0}^{+} \frac{\partial}{\partial x} \phi_{0}^{+}-v_{1}^{-} D_{1}^{-} \frac{\partial}{\partial x} \phi_{1}^{-}+v_{1}^{+} D_{1}^{+} \frac{\partial}{\partial x} \phi_{1}^{+}-v_{2}^{-} D_{2}^{-} \frac{\partial}{\partial x} \phi_{2}^{-}+\cdots-v_{N+1}^{-} D_{N+1}^{-} \frac{\partial}{\partial x} \phi_{N+1}^{-} \\
& =v_{0}^{+} D_{0}^{+} \frac{\partial}{\partial x} \phi_{0}^{+}+\sum_{i=1}^{N}\left(v_{i}^{+} D_{i}^{+} \frac{\partial}{\partial x} \phi_{i}^{+}-v_{i}^{-} D_{i}^{-} \frac{\partial}{\partial x} \phi_{i}^{-}\right)-v_{N+1}^{-} D_{N+1}^{-} \frac{\partial}{\partial x} \phi_{N+1}^{-} .
\end{aligned}
$$

Using the identity

$$
v_{i}^{+} D_{i}^{+} \frac{\partial}{\partial x} \phi_{i}^{+}-v_{i}^{-} D_{i}^{-} \frac{\partial}{\partial x} \phi_{i}^{-}=-\frac{1}{2}\left(v_{i}^{+}+v_{i}^{-}\right)\left(D_{i}^{-} \frac{\partial}{\partial x} \phi_{i}^{-}-D_{i}^{+} \frac{\partial}{\partial x} \phi_{i}^{+}\right)-\frac{1}{2}\left(v_{i}^{-}-v_{i}^{+}\right)\left(D_{i}^{+} \frac{\partial}{\partial x} \phi_{i}^{+}+D_{i}^{-} \frac{\partial}{\partial x} \phi_{i}^{-}\right),
$$

and introducing the notation

$$
\begin{array}{lll}
\{v\}_{i}=\frac{1}{2}\left(v_{i}^{+}+v_{i}^{-}\right), & \left\{D \frac{\partial}{\partial x} \phi\right\}_{i}=\frac{1}{2}\left(D_{i}^{+} \frac{\partial}{\partial x} \phi_{i}^{+}+D_{i}^{-} \frac{\partial}{\partial x} \phi_{i}^{-}\right), \\
{[v]_{i}=v_{i}^{-}-v_{i}^{+},} & {\left[D \frac{\partial}{\partial x} \phi\right]_{i}=D_{i}^{-} \frac{\partial}{\partial x} \phi_{i}^{-}-D_{i}^{+} \frac{\partial}{\partial x} \phi_{i}^{+},}
\end{array}
$$

the term (16), is rewritten as

$$
T(\phi)=v_{0}^{+} D_{0}^{+} \frac{\partial}{\partial x} \phi_{0}^{+}-\sum_{i=1}^{N}\left(\{v\}_{i}\left[D \frac{\partial}{\partial x} \phi\right]_{i}\right)-\sum_{i=1}^{N}\left([v]_{i}\left\{D \frac{\partial}{\partial x} \phi\right\}_{i}\right)-v_{N+1}^{-} D_{N+1}^{-} \frac{\partial}{\partial x} \phi_{N+1}^{-} .
$$

We are looking for solutions of the neutron diffusion equation that satisfy the continuity of the neutron current in the interior vertices of the mesh and that satisfy also the discontinuity factors condition in these vertices. That is,

$$
\begin{aligned}
& {\left[D \frac{\partial}{\partial x} \phi\right]_{i}=0,} \\
& {[\phi]_{f i}=f_{i}^{-} \phi_{i}^{-}-f_{i}^{+} \phi_{i}^{+}=0, i=1,2, \ldots, N,}
\end{aligned}
$$

where $f_{i}^{+}$and $f_{i}^{-}$are the right and left discontinuity factors for vertex $x_{i}$, respectively.

Using the continuity condition for the current in the interior vertices from equation (17), the term (16) can be expressed as

$$
T(\phi)=v_{0}^{+} D_{0}^{+} \frac{\partial}{\partial x} \phi_{0}^{+}-\sum_{i=1}^{N}\left([v]_{i}\left\{D \frac{\partial}{\partial x} \phi\right\}_{i}\right)-v_{N+1}^{-} D_{N+1}^{-} \frac{\partial}{\partial x} \phi_{N+1}^{-},
$$

while preserving the consistency of the formulation (the weak formulation is satisfied by the solution of the original strong formulation defined by equation (10)).

To stabilize this method, a penalty term is introduced (Brezzi et al., 2006),

$$
P(\phi):=\sum_{i=1}^{N} s_{i}\left([\phi]_{f i}[v]_{i}\right)
$$

which does not affect the consistency of the formulation $(P(\phi)=0$ when $\phi$ is satisfies the discontinuity factors condition (18)), and the new term $T^{s p}(\phi):=T(\phi)+P(\phi)$ is written as

$$
T^{s p}(\phi)=v_{0}^{+} D_{0}^{+} \frac{\partial}{\partial x} \phi_{0}^{+}-v_{N+1}^{-} D_{N+1}^{-} \frac{\partial}{\partial x} \phi_{N+1}^{-}-\sum_{i=1}^{N}\left(\left\{D \frac{\partial}{\partial x} \phi\right\}_{i}[v]_{i}\right)+\sum_{i=1}^{N} s_{i}\left([\phi]_{f i}[v]_{i}\right) .
$$


Then, equation (14) is rewritten as

$$
\begin{aligned}
& \sum_{i=0}^{N} \int_{x_{i}}^{x_{i+1}} \frac{\partial v}{\partial x} D \frac{\partial \phi}{\partial x} d x+\sum_{i=0}^{N} \int_{x_{i}}^{x_{i+1}} v \Sigma_{a} \phi d x-\sum_{i=1}^{N}\left(\left\{D \frac{\partial}{\partial x} \phi\right\}_{i}[v]_{i}\right) \\
& +\sum_{i=1}^{N} s_{i}\left([\phi]_{f i}[v]_{i}\right)+v_{0}^{+} D_{0}^{+} \frac{\partial}{\partial x} \phi_{0}^{+}-v_{N+1}^{-} D_{N+1}^{-} \frac{\partial}{\partial x} \phi_{N+1}^{-}=\sum_{i=0}^{N} \int_{x_{i}}^{x_{i+1}} v q d x .
\end{aligned}
$$

Boundary conditions remain to be introduced in the method to be completely defined. The boundary conditions are imposed in a weak form (Rivière, 2008) and this can be made modifying the terms

$$
v_{0}^{+} D_{0}^{+} \frac{\partial}{\partial x} \phi_{0}^{+}-v_{N+1}^{-} D_{N+1}^{-} \frac{\partial}{\partial x} \phi_{N+1}^{-}
$$

in equation (22). Hence, if zero current conditions are imposed, these terms are identically zero. If zero flux boundary conditions are imposed these terms remain unaltered. And if albedo boundary conditions are considered,

$$
-D_{0}^{+} \frac{\partial}{\partial x} \phi_{0}^{+}=\alpha_{0} \phi_{0}^{+}, D_{N+1}^{-} \frac{\partial}{\partial x} \phi_{N+1}^{-}=\alpha_{N+1} \phi_{N+1}^{-},
$$

then, the terms (23) are substituted by

$$
-v_{0}^{+} \alpha_{0} \phi_{0}^{+}-v_{N+1}^{-} \alpha_{N+1} \phi_{N+1}^{-}
$$

This scheme requires the user to specify a parameter, $s_{i}$, which is known as a penalty parameter. This parameter can be viewed as a Lagrange multipliers condition to enforce the discontinuity factor condition in the finite element formulation. If the value of this parameter is not sufficiently large, the approximate solution is unstable. On the other hand, for an arbitrarily large value of the penalty parameter the condition number for the resulting matrices of the discretization would degenerate (Shahbazi, 2005). The development of optimal lower bounds for this parameters is out of the scope of this work, so here the penalty parameters are chosen, based in the expressions provided by Epshteyn and Rivière (2007) and Shahbazi (2005), as a large enough parameter ensuring convergence for the method.

Using the discontinuity factor condition (18), also a symmetrizing term could be introduced in the method, which is identically zero for the exact solution of the problem,

$$
\theta \sum_{i=1}^{N}\left([\phi]_{f i}\left\{D \frac{\partial}{\partial x} v\right\}_{i}\right)
$$

thus equation (22) can be substituted by

$$
\begin{aligned}
& \sum_{i=0}^{N} \int_{x_{i}}^{x_{i+1}} \frac{\partial v}{\partial x} D \frac{\partial \phi}{\partial x} d x+\sum_{i=0}^{N} \int_{x_{i}}^{x_{i+1}} v \Sigma_{a} \phi d x \\
& -\sum_{i=1}^{N}\left(\left\{D \frac{\partial}{\partial x} \phi\right\}_{i}[v]_{i}\right)+\theta \sum_{i=1}^{N}\left([\phi]_{f i}\left\{D \frac{\partial}{\partial x} v\right\}_{i}\right)+\sum_{i=1}^{N} s_{i}\left([\phi]_{f i}[v]_{i}\right) \\
& +v_{0}^{+} D_{0}^{+} \frac{\partial}{\partial x} \phi_{0}^{+}-v_{N+1}^{-} D_{N+1}^{-} \frac{\partial}{\partial x} \phi_{N+1}^{-}=\sum_{i=0}^{N} \int_{x_{i}}^{x_{i+1}} v q d x,
\end{aligned}
$$

where different values for the parameter $\theta=\{-1,0,1\}$ can be selected, obtaining different methods (Sun and Wheeler, 2005). Selecting $\theta=-1$ a method similar to the Symmetric Interior Penalty Galerkin (SIPG) method is obtained. For $\theta=1$ it gives the analogous to the Non-symmetric Interior Penalty Galerkin (NIPG) method, and $\theta=0$ corresponds to the Incomplete Interior Penalty Galerkin (IIPG) method. The symmetrizing term (25) is different from the one used in interior penalty methods, because it has the jump with respect to the discontinuity factors instead of the classical jump respect to the continuity of the solution. Thus, it does not provide symmetry for the linear systems when using the SIPG method, and has not known advantages for the NIPG method. because no known reason justifies using one of the other methods, $\theta=0$ is considered in our formulation. 


\subsection{Multidimensional problems}

The interior penalty finite element method can be generalized to problems with multidimensional geometry. For this kind of problems, equation (10) is written as

$$
-\vec{\nabla} D \vec{\nabla} \phi+\Sigma_{a} \phi=q .
$$

This equation is defined for a reactor $\Omega$, which is divided into subdomains $\Omega_{1}, \Omega_{2}, \ldots, \Omega_{N}$, where the homogenized cross sections are considered as constant values.

Multiplying equation (27) by a test function, $v$, and integrating over $\Omega$, it is obtained

$$
-\int_{\Omega} v \vec{\nabla} D \vec{\nabla} \phi d \vec{r}+\int_{\Omega} v \Sigma_{a} \phi d \vec{r}=\int_{\Omega} v q d \vec{r},
$$

which can be rewritten as

$$
-\sum_{e=1}^{N} \int_{\Omega_{e}} v \vec{\nabla} D \vec{\nabla} \phi d \vec{r}+\int_{\Omega} v \Sigma_{a} \phi d \vec{r}=\int_{\Omega} v q d \vec{r}
$$

Integrating by parts the first term and using Green's theorem, we rewrite the first term of equation (28) as

$$
-\int_{\Omega_{e}} \nu \vec{\nabla} D \vec{\nabla} \phi d \vec{r}=D_{e} \int_{\Omega_{e}} \vec{\nabla} \nu \vec{\nabla} \phi d \vec{r}-\int_{\partial \Omega_{e}} \nu D \vec{\nabla} \phi \vec{n} d S .
$$

In this way, equation (28), can be written as

$$
\sum_{e=1}^{N} D_{e} \int_{\Omega_{e}} \vec{\nabla} v \vec{\nabla} \phi d \vec{r}-\sum_{e=1}^{N} \int_{\partial \Omega_{e}} v D \vec{\nabla} \phi \vec{n} d S+\int_{\Omega} v \Sigma_{a} \phi d \vec{r}=\int_{\Omega} v q d \vec{r}
$$

The surface integrals are decomposed as a sum over the different faces limiting each node, distinguishing the interior faces $E_{1}, E_{2}, \ldots, E_{I}$ from the faces, $B_{1}, B_{2}, \ldots, B_{F}$, placed at the boundary of the reactor. For two adjacent nodes $\Omega_{i}$ and $\Omega_{j}$, sharing the interior face $E_{l}$, the jump and average operators are defined by

$$
\begin{array}{lll}
\{D \vec{\nabla} \phi\}_{E_{l}}=\frac{1}{2}\left(D_{i} \vec{\nabla} \phi_{i}^{-}+D_{j} \vec{\nabla} \phi_{j}^{+}\right), & \{v\}_{E_{l}}=\frac{1}{2}\left(v_{i}^{-}+v_{j}^{+}\right), \\
{[D \vec{\nabla} \phi]_{E_{l}}=D_{i} \vec{\nabla} \phi_{i}^{-} \vec{n}_{i}+D_{j} \vec{\nabla} \phi_{j}^{+} \vec{n}_{j},} & {[v]_{E_{l}}=v_{i}^{-} \vec{n}_{i}+v_{j}^{+} \vec{n}_{j},}
\end{array}
$$

where $D_{i}, \phi_{i}^{ \pm}, v_{i}^{ \pm}$are the lateral limits in the surface $E_{l}$ of $D, \phi, v$ in the node $\Omega_{i}$ and $\vec{n}_{i}$ is the normal vector to the face $E_{l}$ pointing outwards of node $\Omega_{i}$. Collecting together the integrals over the same face and operating in a similar way as it has been done for the one-dimensional case, using the definitions (30), we set

$$
-\sum_{e=1}^{N} \int_{\partial \Omega_{e}} \nu D \vec{\nabla} \phi \vec{n} d S=-\sum_{j=1}^{F} \int_{B_{j}} \nu D \vec{\nabla} \phi \vec{n} d S-\sum_{l=1}^{I} \int_{E_{l}}\{D \vec{\nabla} \phi\}_{E_{l}}[v]_{E_{l}} \vec{n} d S-\sum_{l=1}^{I} \int_{E_{l}}[D \vec{\nabla} \phi]_{E_{l}}\{v\}_{E_{l}} \vec{n} d S .
$$

We are looking for a solution, $\phi$, of the neutron diffusion equation (27) on each of the subdomains $\Omega_{e}$ such that in each interior surface, $E_{l}$ that is shared by nodes $\Omega_{i}$ and $\Omega_{j}$, the neutronic current is continuous and the assembly discontinuity factors condition is satisfied. These conditions can be expressed as

$$
\begin{aligned}
& {[D \vec{\nabla} \phi]_{E_{l}}=0,} \\
& {[\phi]_{f_{E_{l}}}=f_{i}^{-} \phi_{i} \vec{n}_{i}+f_{j}^{+} \phi_{j} \vec{n}_{j}=0,}
\end{aligned}
$$


which are imposed in a weak form in the interior penalty method.

Using the current continuity condition (32), equation (31) is rewritten as

$$
-\sum_{e=1}^{N} \int_{\Omega_{e}} v D \vec{\nabla} \phi \vec{n} d S=-\sum_{j=1}^{F} \int_{B_{j}} v D \vec{\nabla} \phi \vec{n} d S-\sum_{l=1}^{I} \int_{E_{l}}\{D \vec{\nabla} \phi\}_{E_{l}}[v]_{E_{l}} \vec{n} d S .
$$

As in the one-dimensional case, using the assembly discontinuity factors condition (33), a penalty term is introduced, thus,

$$
-\sum_{e=1}^{N} \int_{\Omega_{e}} v D \vec{\nabla} \phi d \vec{r}=-\sum_{j=1}^{F} \int_{B_{j}} v D \vec{\nabla} \phi \vec{n} d S-\sum_{l=1}^{I} \int_{E_{l}}\{D \vec{\nabla} \phi\}_{E_{l}}[v]_{E_{l}} \vec{n} d S+\sum_{l=1}^{I} \int_{E_{l}} s_{l}[\phi]_{f_{E_{l}}}[v]_{E_{l}} d S .
$$

Inserting the expression (35) into equation (29) the multidimensional interior penalty method for the neutron diffusion equation is obtained.

$$
\begin{aligned}
& \sum_{e=1}^{N} D_{e} \int_{\Omega_{e}} \vec{\nabla} v \vec{\nabla} \phi d \vec{r}+\int_{\Omega} v \Sigma_{a} \phi d \vec{r}-\sum_{l=1}^{I} \int_{E_{l}}\{D \vec{\nabla} \phi\}_{E_{l}}[v]_{E_{l}} \vec{n} d S \\
& +\sum_{l=1}^{I} \int_{E_{l}} s_{l}[\phi]_{E_{E_{l}}}[v]_{E_{l}} \vec{n} d S-\sum_{j=1}^{F} \int_{B_{j}} v D \vec{\nabla} \phi \vec{n} d S=\int_{\Omega} v q d \vec{r} .
\end{aligned}
$$

The boundary conditions in the method affect the surface integrals over the external faces $B_{j}$ and the changes are similar to the ones exposed in the one-dimensional case.

The penalty parameters $s_{l}$ are chosen as a large enough parameter ensuring convergence for the method, based in the expressions provided by (Epshteyn and Rivière, 2007) and (Shahbazi, 2005).

As in the one-dimensional case, using the discontinuity factors condition (33), a symmetrizing term could be introduced, obtaining

$$
\begin{aligned}
& \sum_{e=1}^{N} D_{e} \int_{\Omega_{e}} \vec{\nabla} v \vec{\nabla} \phi d \vec{r}+\int_{\Omega} v \Sigma_{a} \phi d \vec{r} \\
& -\sum_{l=1}^{I} \int_{E_{l}}\{D \vec{\nabla} \phi\}_{E_{l}}[v]_{E_{l}} \vec{n} d S+\theta \sum_{l=1}^{I} \int_{E_{l}}[\phi]_{f_{E_{l}}}\{D \vec{\nabla} v\}_{E_{l}} \vec{n} d S \\
& +\sum_{l=1}^{I} \int_{E_{l}} s_{l}[\phi]_{E_{E_{l}}}[v]_{E_{l}} d S-\sum_{j=1}^{F} \int_{B_{j}} v D \vec{\nabla} \phi \vec{n} d S=\int_{\Omega} v q d \vec{r} .
\end{aligned}
$$

Nevertheless, for the same reason as in one-dimensional problems, no symmetrizing term is considered in our formulation $(\theta=0)$.

\section{Reference homogenized cross sections and discontinuity factors}

In order to calculate the best possible homogenized solution, the heterogeneous solution over the whole reactor is used to calculate the reference values of the homogenized coefficients, cross sections and discontinuity factors. The computed parameters are called reference homogenized cross sections (RXS) and reference discontinuity factors (RDFs) to differentiate them from the isolated assembly parameters, assembly homogenized cross sections (AXS) and assembly discontinuity factors (ADFs), which are computed using the heterogeneous flux in an isolated assembly 
with reflective boundary conditions. Obviously, this reference homogenized calculation does not have any practical application because the full heterogeneous reactor is must be solved first. However, the reference homogenized solution can be used to distinguish between the error when solving the homogenized problem, and the error of the homogenization procedure.

To compute the reference cross sections the neutron diffusion equation is solved taking into account the heterogeneous composition of each one of the assemblies composing the reactor, obtaining the heterogeneous neutron flux, $\phi_{g}(\vec{r})$. Reference cross sections have been calculated with the usual flux-weighting method by means of equation (4) and (7) (Smith, 1986). A calculation method of the reference discontinuity factors has been proposed in one dimensional assemblies and its extension to multidimensional assemblies.

\subsection{One-dimensional assemblies}

First, the solution for the heterogeneous whole core reactor is obtained, $\phi_{g}(x)$. Using this flux, the reference homogenized cross sections, $\hat{\Sigma}_{\alpha, g}$ and $\hat{D}_{g}$ are obtained for the different assemblies. The reference discontinuity factors at vertex $x_{i}$ are defined as

$$
f_{g, i}^{-}=\frac{\phi_{g}^{-}\left(x_{i}\right)}{\hat{\phi}_{g}^{-}\left(x_{i}\right)}, \quad f_{g, i-1}^{+}=\frac{\phi_{g}^{+}\left(x_{i}\right)}{\hat{\phi}_{g}^{+}\left(x_{i}\right)},
$$

depending also on the homogenized flux. This homogenized flux is the solution of a boundary value problem, defined in one-group of energy (see Figure 3) as

$$
-\hat{D}_{i} \frac{\mathrm{d}^{2}}{\mathrm{~d} x^{2}} \hat{\phi}(x)+\hat{\Sigma}_{a, i} \hat{\phi}(x)-\frac{1}{k_{\mathrm{eff}}} v \hat{\Sigma}_{f, i} \hat{\phi}(x)=0
$$

with the boundary conditions

$$
\begin{aligned}
-\hat{D}_{i} \frac{\mathrm{d} \hat{\phi}}{\mathrm{d} x}\left(x_{i}\right) & =\hat{J}_{x}\left(x_{i}\right), \\
-\hat{D}_{i} \frac{\mathrm{d} \hat{\phi}}{\mathrm{d} x}\left(x_{i+1}\right) & =\hat{J}_{x}\left(x_{i+1}\right) .
\end{aligned}
$$

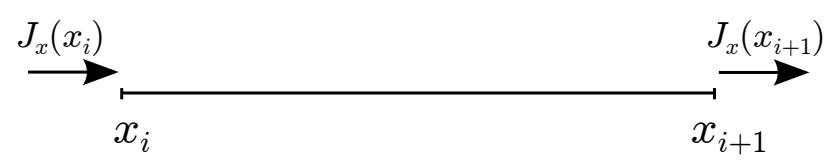

Figure 3: 1D assembly.

These boundary conditions and the $k_{\text {eff }}$ present in equation (39) are obtained from the whole core heterogeneous reactor calculation, using

$$
\begin{aligned}
& \hat{J}_{x}\left(x_{i}\right) \approx J_{x}\left(x_{i}\right)=D_{i} \frac{\mathrm{d} \phi}{\mathrm{d} x}\left(x_{i}\right), \\
& \hat{J}_{x}\left(x_{i+1}\right) \approx J_{x}\left(x_{i+1}\right)=D_{i} \frac{\mathrm{d} \phi}{\mathrm{d} x}\left(x_{i+1}\right) .
\end{aligned}
$$

Although, problem (39) has an analytical solution for one energy group approximation, the solution of the same problem for the multigroup approximation has to be computed numerically. The numerical method selected to solve 
this problem has been a high-order continuous finite element method (Vidal-Ferrandiz et al., 2014). The weak form of this problem for a test function $\varphi$ can be written as

$$
\hat{D}_{i} \int_{x_{i}}^{x_{i+1}} \frac{\mathrm{d} \varphi}{\mathrm{d} x} \frac{\mathrm{d} \hat{\phi}}{\mathrm{d} x} \mathrm{~d} x+\left(\hat{\Sigma}_{a, i}-\frac{v \hat{\Sigma}_{f, i}}{k_{\text {eff }}}\right) \int_{x_{i}}^{x_{i+1}} \varphi \hat{\phi} \mathrm{d} x=\varphi \hat{J}_{x}\left(x_{i}\right)-\varphi \hat{J}_{x}\left(x_{i+1}\right)
$$

which after spatial discretization becomes the algebraic linear system

$$
A \Phi=f
$$

where $\Phi$ is the vector of coefficients of of the polynomial expansion of the homogeneous neutron flux. The elements of the matrix $A$ and the right hand side vector $f$ are given by

$$
\begin{aligned}
A_{l m} & =\hat{D}_{i} \int_{x_{i}}^{x_{i+1}} \frac{\mathrm{d} N_{l}}{\mathrm{~d} x} \frac{\mathrm{d} N_{m}}{\mathrm{~d} x} \mathrm{~d} x+\left(\hat{\Sigma}_{a i}-\frac{v \hat{\Sigma}_{f, i}}{k_{\mathrm{eff}}}\right) \int_{x_{i}}^{x_{i+1}} N_{l} N_{m} \mathrm{~d} x, \\
f_{l} & =\left\{\begin{array}{cl}
+N_{l} \hat{J}_{x}\left(x_{i}\right) & \text { if } l=0, \\
0 & \text { if } l \neq 0, K, \\
-N_{l} \hat{J}_{x}\left(x_{i+1}\right) & \text { if } l=K .
\end{array}\right.
\end{aligned}
$$

where $N_{l}$ are the shape function considered and $K$ is the polynomial truncation order chosen.

\subsection{Multidimensional assemblies}

The method exposed above, used to obtain the homogeneous flux contributing to the discontinuity to the discontinuity factors definition, can be generalized to multidimensional assemblies by alternatively solving coupled onedimensional problems following the lines presented in Smith (1986). To present the method, a two-dimensional assembly, like the one shown in Figure 4, is considered.

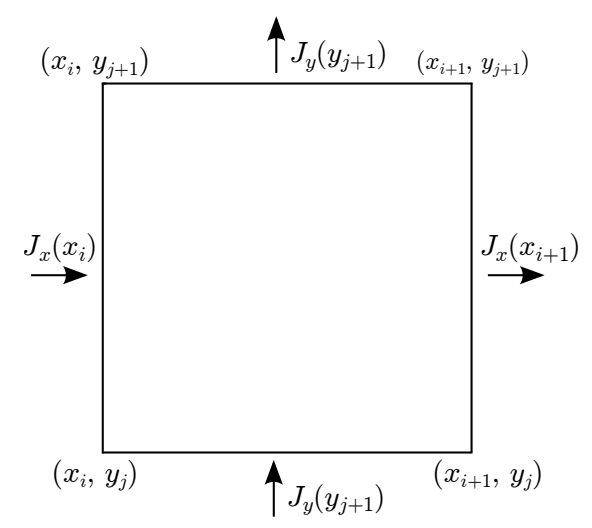

Figure 4: 2D assembly.

The Reference Discontinuity Factors for the faces containing the vertices $\left(x_{i}, y_{j}\right)$ and $\left(x_{i}, y_{j+1}\right)$ (parallel to the $y$ axis) are defined by

$$
f_{g, i}^{-}=\frac{\int_{y_{j}}^{y_{j+1}} \phi_{g}^{-}\left(x_{i}, y\right) \mathrm{d} y}{\int_{y_{j}}^{y_{j+1}} \hat{\phi}_{g}^{-}\left(x_{i}, y\right) \mathrm{d} y} ; \quad f_{g, i-1}^{+}=\frac{\int_{y_{j}}^{y_{j+1}} \phi_{g}^{+}\left(x_{i}, y\right) \mathrm{d} y}{\int_{y_{j}}^{y_{j+1}} \hat{\phi}_{g}^{+}\left(x_{i}, y\right) \mathrm{d} y} .
$$


The definition of the discontinuity factors for the other faces of the assembly (parallel to the $x$ axis) is similar. In order to calculate the discontinuity factors, the homogenized flux has to be calculated. Lets consider the neutron diffusion equation for the 2D assembly $i$

$$
-\vec{\nabla} \hat{D}_{i} \vec{\nabla} \hat{\phi}(x, y)+\Sigma_{a, i} \hat{\phi}(x, y)=v \Sigma_{f, i} \hat{\phi}(x, y) .
$$

Integrating along the $y$ direction, equation (43) can be written as the one-dimensional problem

$$
-\hat{D}_{i} \frac{\mathrm{d}^{2}}{\mathrm{~d} x^{2}} \hat{\Phi}(x)+\left(\hat{\Sigma}_{a, i}-\frac{v \hat{\Sigma}_{f, i}}{k_{\mathrm{eff}}}\right) \hat{\Phi}(x)=S(x),
$$

where the unknown, $\hat{\Phi}(x)$, is defined by

$$
\hat{\Phi}(x)=\int_{y_{i}}^{y_{i+1}} \hat{\phi}(x, y) \mathrm{d} y,
$$

and the leakage

$$
S(x)=\hat{D}_{i} \int_{y_{i}}^{y_{i+1}} \frac{\mathrm{d}^{2}}{\mathrm{~d} y^{2}} \hat{\phi}(x, y) \mathrm{d} y,
$$

has been introduced, which is computed using the previously calculated heterogeneous neutronic flux. The boundary conditions considered for this problem are as follows

$$
\begin{gathered}
\hat{J}_{x}\left(x_{i}\right)=-\hat{D}_{i} \frac{\mathrm{d}}{\mathrm{d} x} \hat{\Phi}\left(x_{i}\right)=\int_{y_{j}}^{y_{j+1}} \frac{\mathrm{d}}{\mathrm{d} x} \phi\left(x_{i}, y\right) \mathrm{d} y, \\
\hat{J}_{x}\left(x_{i+1}\right)=-\hat{D}_{i} \frac{\mathrm{d}}{\mathrm{d} x} \hat{\Phi}\left(x_{i+1}\right)=\int_{y_{j}}^{y_{j+1}} \frac{\mathrm{d}}{\mathrm{d} x} \phi\left(x_{i+1}, y\right) \mathrm{d} y,
\end{gathered}
$$

which are also obtained from the heterogeneous neutron flux.

The resulting problem is a one-dimensional source problem similar to the one exposed in subsection 4.1, which is solved using the finite element method. A similar procedure is used to obtain the Reference Discontinuity Factors in the other faces of the assembly (parallel to the $y$ axis).

\section{Numerical Results}

To assess the capabilities of the proposed interior penalty method with assembly discontinuity factors, a code has been implemented in a standard finite element library named Deal.II (Bangerth et al., 2007) using Lagrange polynomials.

To check the performance of the discontinuous Galerkin method in introducing the discontinuity factor conditions between the interior faces of adjacent nodes, first we will use the reference cross sections (RXS) and reference assembly discontinuity factors (RDF) computed from the heterogeneous flux. These results are complemented with the calculations using the homogenized cross sections (AXS) and the assembly discontinuity factors (ADF), calculated considering an isolated assembly with reflecting boundary conditions during the homogenization process.

Several one and two-dimensional benchmark problems have been analysed and the results obtained with the finite element method have been compared with the results obtained with the code PARCS (Joo et al., 1998). The 1-D benchmarks are slab of assemblies which are simplified representations of Boiling Water Reactor (BWR) configurations, while the 2-D benchmark problems are two small subsets of the CISE core and the complete CISE core (Rahnema and Nichita, 1997).

\subsection{One dimensional problems}

The 1D cores are extracted from (Rahnema and Nichita, 1997) and are composed of seven assemblies. These configurations are constructed by combining three different assemblies which are 1-D representations of BWR assemblies 
(Chiang, 1982). The geometry of the assemblies and their configurations are shown in Figure 5. Cross sections for each region of these assemblies are given in Table 1. Configurations are sort by heterogeneities between assemblies. The first configuration is composed of similar assemblies and in the last one heterogeneous assemblies have been joined together, which is used to test the method in an extreme situation. Zero-current boundary conditions are imposed at the edges of the system. All the calculation are made using cubic polynomials in the finite element method.

\section{Configuration 1}

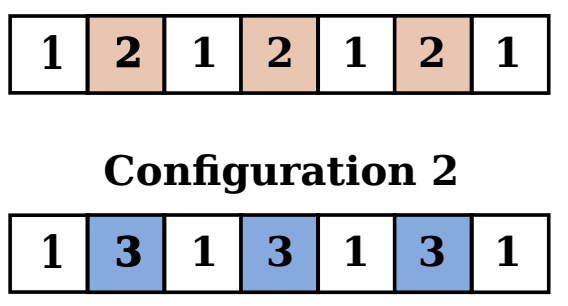

Configuration 3

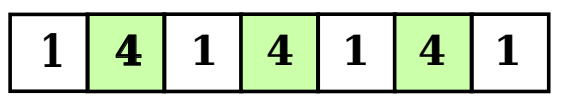

Type 1 Type 2

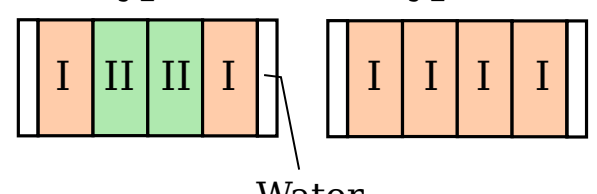

Water

Type 3

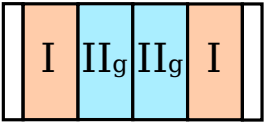

Type 4

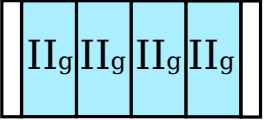

Figure 5: Core and assemblies configurations for the 1D benchmark problems.

Table 1: Material properties for the 1D benchmark problems.

\begin{tabular}{lcccccccc}
\hline \hline Mat. & $\begin{array}{c}\text { Thickness } \\
(\mathrm{cm})\end{array}$ & $\begin{array}{c}\mathbf{D}_{\mathbf{1}} \\
(\mathrm{cm})\end{array}$ & $\begin{array}{c}\mathbf{D}_{\mathbf{2}} \\
(\mathrm{cm})\end{array}$ & $\begin{array}{c}\boldsymbol{\Sigma}_{\boldsymbol{a} \mathbf{1}} \\
(1 / \mathrm{cm})\end{array}$ & $\begin{array}{c}\boldsymbol{\Sigma}_{\boldsymbol{a} \mathbf{2}} \\
(1 / \mathrm{cm})\end{array}$ & $\begin{array}{c}\boldsymbol{\Sigma}_{\mathbf{1 2}} \\
(1 / \mathrm{cm})\end{array}$ & $\begin{array}{c}\boldsymbol{v} \boldsymbol{\Sigma}_{\boldsymbol{f} \mathbf{1}} \\
(1 / \mathrm{cm})\end{array}$ & $\begin{array}{c}\boldsymbol{v} \boldsymbol{\Sigma}_{\boldsymbol{f} \mathbf{2}} \\
(1 / \mathrm{cm})\end{array}$ \\
\hline Water & 1.158 & 1.7639 & 0.2278 & 0.0003 & 0.0097 & 0.0380 & 0.0000 & 0.0000 \\
Fuel I & 3.231 & 1.4730 & 0.3294 & 0.0096 & 0.0764 & 0.0161 & 0.0067 & 0.1241 \\
Fuel II & 3.231 & 1.4804 & 0.3362 & 0.0101 & 0.0901 & 0.0156 & 0.0078 & 0.1542 \\
Fuel IIg & 3.231 & 1.5342 & 0.3143 & 0.0135 & 0.4873 & 0.0136 & 0.0056 & 0.0187 \\
\hline \hline
\end{tabular}

In addition to the explained finite element calculations, two more cases are presented for comparative purposes. The first one is done with the nodal code PARCS (Joo et al., 1998) using discontinuity factors. All PARCS calculations are made with 16 cells per assembly in order to achieve a reasonable accuracy for these small problems. The reference solution is obtained using an heterogeneous fine mesh calculation made with the finite element code.

Assembly errors are condensed using the root mean square (RMS) of the relative errors as defined in equation (45)

$$
\mathrm{RMS}=\sqrt{\frac{1}{\sum_{i} V_{i}} \sum_{i} V_{i}\left(\frac{\varphi_{\text {hom }, i}-\varphi_{\text {het }, i}}{\varphi_{\text {het }, i}}\right)^{2}},
$$

where $\varphi_{\text {hom }, i}$ and $\varphi_{\text {het }, i}$ represent the homogeneous and the heterogeneous (reference) variable of interest (either fluxes or power averaged in assembly $i$ ). $V_{i}$ represent the measure of the region $i$ (longitude in 1D and area in 2D).

Figure 6 shows the fast and thermal fluxes for the homogenized reactor associated with Configuration 1, making use of the discontinuity factors and without using them. It is shown that a discontinuous solution for the flux is obtained with the interior penalty finite element method proposed here. 

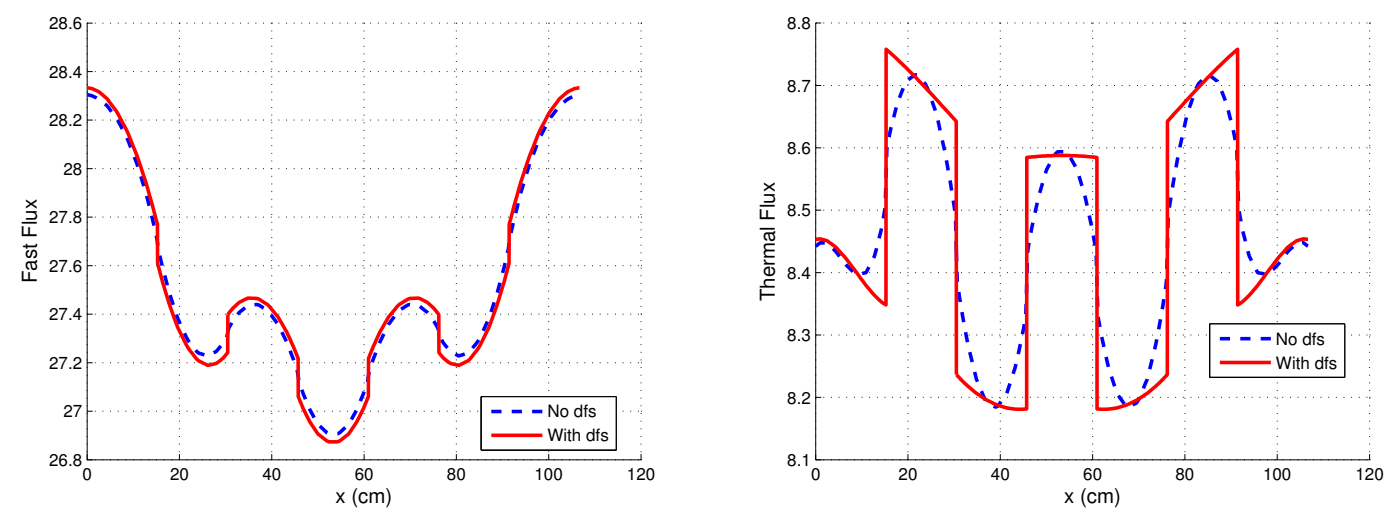

Figure 6: Fast and thermal homogeneous fluxes for the Configuration 1.

System $k_{\text {eff }}$ and assembly averaged fluxes and power errors for configurations 1, 2 and 3 are given in Tables 2, 3, and 4, respectively. In Table 2 it is shown a very good behavior for all the methods, being the solution with the RXS and RDFs the most accurate. It is worth to notice that this problem represents the less heterogeneous configuration, so the solution without discontinuity factors, even if the worst one, is obtained with very good accuracy.

Table 2: Results for Configuration 1 core.

\begin{tabular}{lccccc}
\hline \hline & & \multicolumn{4}{c}{ Assembly Errors (\%) } \\
Method & $k_{\text {eff }}$ & $\begin{array}{c}\text { Error } \\
\text { Fcm) }\end{array}$ & $\begin{array}{c}\text { Fast } \\
\text { Flux }\end{array}$ & $\begin{array}{c}\text { Thermal } \\
\text { Flux }\end{array}$ & Power \\
\hline FEM (No DFs) & 1.31997 & 5 & 0.19 & 0.47 & 0.36 \\
FEM (DFs) & 1.31991 & 1 & 0.04 & 0.02 & 0.03 \\
PARCS (DFs) & 1.31990 & 2 & 0.05 & 0.04 & 0.04 \\
FEM (RDFs) & 1.31992 & 0 & 0.02 & 0.04 & 0.04 \\
Heterogeneous & 1.31992 & & & & \\
\hline \hline
\end{tabular}

Table 3 shows the results for a more heterogeneous configuration. For this problem, the use of discontinuity factors become necessary. We can observe a great improvement of the $k_{\text {eff }}$ approximation, even if the errors in the flux are mostly the same as without discontinuity factors. Again, a good agreement is found between the finite element method with ADFs and PARCS with ADFs. For this reactor the RXS and RDFs the most accurate, meaning that the homogenization process should be improved to achieve higher accuracy.

Table 3: Results for Configuration 2 core.

\begin{tabular}{|c|c|c|c|c|c|}
\hline \multirow[b]{2}{*}{ Method } & \multirow[b]{2}{*}{$k_{\mathrm{eff}}$} & \multirow[b]{2}{*}{$\begin{array}{l}\text { Error } \\
(\mathrm{pcm})\end{array}$} & \multicolumn{3}{|c|}{ Assembly Errors(\%) } \\
\hline & & & $\begin{array}{l}\text { Fast } \\
\text { Flux }\end{array}$ & $\begin{array}{l}\text { Thermal } \\
\text { Flux }\end{array}$ & Power \\
\hline FEM (No DFs) & 1.10350 & 1736 & 3.25 & 3.19 & 3.83 \\
\hline FEM (ADFs) & 1.11934 & 152 & 1.31 & 5.90 & 3.58 \\
\hline PARCS (ADFs) & 1.11955 & 131 & 1.32 & 6.03 & 3.69 \\
\hline FEM (RDFs) & 1.12062 & 14 & 0.10 & 0.18 & 0.16 \\
\hline Heterogeneous & 1.12086 & & & & \\
\hline
\end{tabular}

For Configuration 3, the most heterogeneous among the ones chosen for the one-dimensional problems, we observed 
that more accurate calculations (higher order polynomial expansions for the FEM) are necessary in order to converge the method, so 5th order degree FEM has been added to the calculations. Using ADFs, we see that the FEM converges, when increasing the truncation degree, to the same solution as PARCS with ADF. Using the RDFs it is also necessary to go to 5th degree to achieve accurate results. This is due to the strong heterogeneity of the reactor, what makes also more challenging for the Finite Element Method to converge spatially (the homogenization error is larger for this configuration, but also the error due to the FEM approximation).

Table 4: Results for Configuration 3 core.

\begin{tabular}{lccrrr}
\hline \hline & & \multicolumn{4}{c}{ Assembly Errors(\%) } \\
Method & $k_{\text {eff }}$ & $\begin{array}{r}\text { Error } \\
(\mathrm{pcm})\end{array}$ & $\begin{array}{r}\text { Fast } \\
\text { Flux }\end{array}$ & $\begin{array}{r}\text { Thermal } \\
\text { Plux }\end{array}$ & \\
\hline FEM (No DFs; 3rd degree polynomials) & 1.01482 & 703 & 4.56 & 13.56 & 6.22 \\
FEM (No DFs; 5th degree polynomials) & 1.01463 & 722 & 4.38 & 13.45 & 6.02 \\
FEM (ADFs; 3rd degree polynomials) & 1.02464 & 279 & 1.68 & 15.23 & 3.31 \\
FEM (ADFs; 5th degree polynomials) & 1.02797 & 612 & 3.15 & 18.18 & 6.80 \\
PARCS (ADFs) & 1.02865 & 680 & 3.42 & 16.97 & 5.25 \\
FEM (RDFs; 3rd degree polynomials) & 1.01828 & 357 & 2.96 & 4.05 & 3.85 \\
FEM (RDFs; 5th degree polynomials) & 1.02131 & 54 & 0.50 & 0.68 & 0.65 \\
Heterogeneous & 1.02185 & & & & \\
\hline \hline
\end{tabular}

\subsection{Multidimensional problems}

The two-dimensional test problems chosen are based in the CISE benchmark (Rahnema and Nichita, 1997). The layout for the CISE core, together with the assembly definition, are shown in Figure 7, while the cross sections for the blade, water, the fuel A (fresh nuclear fuel) and the fuel B (depleted nuclear fuel) used in the tests are given in Table 5.

\begin{tabular}{|c|c|c|c|c|c|c|c|c|}
\hline W & $W$ & W & W & & & & & \\
\hline A & A & A & W & W & W & & & \\
\hline$A$ & B & A & $\mathrm{A}$ & A & W & W & & \\
\hline B & A & B & $B$ & $\mathrm{~A}$ & A & W & W & \\
\hline A & B & A & $\mathrm{B}$ & $\mathrm{A}$ & $\mathrm{A}$ & A & W & \\
\hline B & A & B & $\mathrm{A}$ & B & B & A & W & W \\
\hline A & $\mathrm{B}$ & A & B & $\mathrm{A}$ & B & A & $\mathrm{A}$ & W \\
\hline B & $\mathrm{A}$ & B & A & B & A & B & A & W \\
\hline $\mathrm{A}$ & B & A & B & $\mathrm{A}$ & B & $\mathrm{A}$ & $\mathrm{A}$ & W \\
\hline
\end{tabular}

(a) Quarter of the whole core CISE reactor composition.

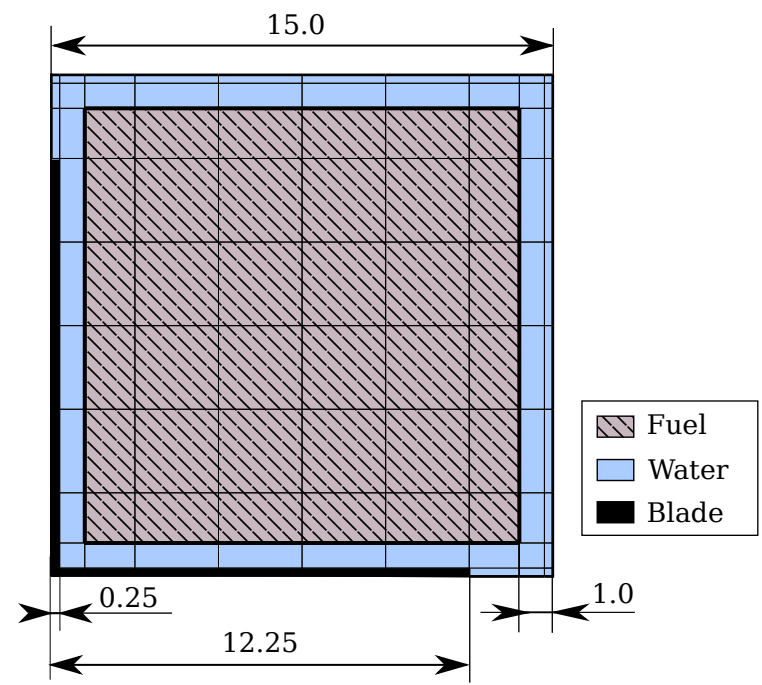

(b) Detailed view of the mesh used for the fine mesh heterogeneous calculation.

Figure 7: CISE core and assembly definition. 
Table 5: Cross section values for CISE benchmarks.

\begin{tabular}{cccccccc}
\hline \hline Material & $\begin{array}{c}\mathbf{D}_{\mathbf{1}} \\
(\mathrm{cm})\end{array}$ & $\begin{array}{c}\mathbf{D}_{\mathbf{2}} \\
(\mathrm{cm})\end{array}$ & $\begin{array}{c}\boldsymbol{\Sigma}_{\boldsymbol{a} \mathbf{1}} \\
(1 / \mathrm{cm})\end{array}$ & $\begin{array}{c}\boldsymbol{\Sigma}_{\boldsymbol{a} \mathbf{2}} \\
(1 / \mathrm{cm})\end{array}$ & $\begin{array}{c}\boldsymbol{\Sigma}_{\mathbf{1 2}} \\
(1 / \mathrm{cm})\end{array}$ & $\begin{array}{c}\boldsymbol{v} \boldsymbol{\Sigma}_{\boldsymbol{f} \mathbf{1}} \\
(1 / \mathrm{cm})\end{array}$ & $\begin{array}{c}\boldsymbol{v} \boldsymbol{\Sigma}_{\boldsymbol{f} \mathbf{2}} \\
(1 / \mathrm{cm})\end{array}$ \\
\hline Water & 2.0000 & 0.3000 & 0.0000 & 0.0100 & 0.0400 & 0.0000 & 0.0000 \\
Fuel A & 1.8000 & 0.5500 & 0.0080 & 0.0850 & 0.0120 & 0.0060 & 0.1100 \\
Fuel B & 1.8000 & 0.5500 & 0.0080 & 0.0850 & 0.0120 & 0.0050 & 0.1000 \\
Blade & 3.0000 & 0.1500 & 0.0800 & 1.0000 & 0.0000 & 0.0000 & 0.0000 \\
\hline \hline
\end{tabular}

Three problems are defined based on the CISE benchmark. First, a small subset composed by four assemblies surrounding an inserted control blade is considered, together with reflective boundary conditions. The geometry for the proposed problem, named Small CISE, is shown in Figure 8a. Within this problem, the heterogeneities considered inside the reactor are taken into account. In order to analyze the method against a more heterogeneous problem, a non-realistic problem, called Modified Small CISE, is defined (see Figure 8b). This problem is similar to the small Cise, but the control blade inside the assemblies defined with material A is removed. This causes the flux to have an strong gradient on the interfaces, thus being more challenging for the homogenization procedure to preserve the properties of the original problem. The third problem to be considered is the original whole core CISE reactor (see Figure 7), together with zero-flux boundary conditions.

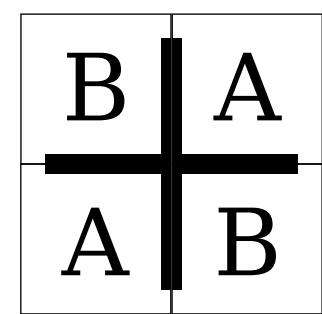

(a) Small CISE

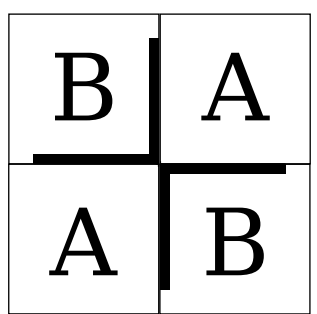

(b) Modified small CISE

Figure 8: Definition of subsets of the CISE reactor

An heterogeneous fine-mesh whole core calculation (Heterogeneous), with the fine mesh shown in Figure $7 \mathrm{~b}$, is used as reference solution. Then, after the homogenization process, the different problems are solved using a coarsemesh of once cell per assembly, together with the different homogenized parameters and discontinuity factors, both the isolated assembly homogenized data (FEM (ADFs)), and the reference homogenized data (FEM (RDFs)). The calculations are complemented with the results obtained by PARCS with the ADFs and AXSs (PARCS (ADFs)), in order to verify the introduction of the discontinuity factors in the FEM. The calculations with the Finite Element code, as in the previous section, were made using cubic polynomials,

The results for the small CISE benchmark are shown in Table 6 . We can see that all the methods show very accurate solution, being the calculations with the RDFs and RXSs the most accurate ones. It is worth to note the agreement between the FEM and PARCS when using ADFs and AXSs, being the error due mostly to the homogenization process, and corrected only by means of an improvement of the homogenized data.

Table 7 shows the results for the modified small CISE problem. This problem has been artificially modified to have stronger heterogeneities. We can observe that the results without using discontinuity factors are very big, while the introduction of the discontinuity factors mitigate the error due to the homogenization process. Comparing the solution of FEM with ADFs and PARCS with ADFs we observe that the two codes agree very well, and these errors can be reduced by means of improving the homogenization procedure, as we can see when we use the RDFs and RXSs. 
Table 6: Results for small CISE benchmark.

\begin{tabular}{lcrccc}
\hline \hline & & \multicolumn{4}{c}{ Assembly Errors(\%) } \\
Method & $k_{\text {eff }}$ & $\begin{array}{r}\text { Error } \\
\text { Fast }\end{array}$ & Thermal & Power \\
& & Flux & Flux & \\
\hline FEM (No DFs) & 0.83189 & 54 & 0.68 & 1.03 & 1.06 \\
FEM (ADFs) & 0.83188 & 55 & 0.71 & 1.06 & 1.09 \\
PARCS (ADFs) & 0.83184 & 59 & 0.72 & 1.06 & 1.09 \\
FEM (RDFs) & 0.83249 & 6 & 0.16 & 0.13 & 0.13 \\
Heterogeneous & 0.83243 & & & & \\
\hline \hline
\end{tabular}

Table 7: Result for the modified small CISE.

\begin{tabular}{lcrccc}
\hline \hline \multirow{2}{*}{ Method } & \multicolumn{5}{c}{ Assembly Errors(\%) } \\
& $k_{\text {eff }}$ & $\begin{array}{r}\text { Error } \\
\text { (pcm) }\end{array}$ & Fast & Thermal & Power \\
& & Flux & \\
\hline FEM (No DFs) & 0.94627 & 5354 & 6.37 & 11.32 & 7.90 \\
FEM (ADFs) & 0.92747 & 3473 & 4.06 & 4.53 & 5.37 \\
PARCS (ADFs) & 0.92754 & 3480 & 4.06 & 4.45 & 5.29 \\
FEM (RDFs) & 0.88843 & 431 & 1.58 & 2.54 & 2.35 \\
Heterogeneous & 0.89274 & & & & \\
\hline \hline
\end{tabular}

Table 8 shows the results for the whole CISE reactor problem. The main differences between this problem and the previous ones is that here we use zero-flux boundary conditions, and that we have some assemblies (defining one layer of reflector) composed only by moderator. The use of zero-flux boundary conditions produce higher flux gradients close to the reflector, making the problem more challenging and thus requiring increase the polynomial order of the FEM. Moreover, reflector assemblies should be considered inside a macro-assembly while homogenizing the surrounding assemblies during the isolated assembly homogenization process, otherwise the homogenization error close to the reflector will also be higher. It has not been done here, but instead the RXSs and RDFs take them into account, so the homogenization error can be reduced. It is observed that, even if the assembly errors are around 5\%

Table 8: Results for CISE benchmark.

\begin{tabular}{lrrrrr}
\hline \hline & & \multicolumn{4}{c}{ Assembly Errors(\%) } \\
Method & $k_{\text {eff }}$ & $\begin{array}{r}\text { Error } \\
\text { Fast }\end{array}$ & $\begin{array}{r}\text { Fast } \\
\text { Flux }\end{array}$ & Flux & Power \\
& & pcmal & \\
\hline FEM (No DFs; 3rd degree polynomials) & 0.94957 & 192 & 5.76 & 4.33 & 5.10 \\
FEM (No DFs; 5th degree polynomials) & 0.94957 & 192 & 5.69 & 4.30 & 5.07 \\
FEM (ADFs; 3rd degree polynomials) & 0.95100 & 49 & 0.79 & 2.00 & 1.07 \\
FEM (ADFs; 5th degree polynomials) & 0.95092 & 57 & 0.89 & 2.11 & 1.15 \\
PARCS (ADFs) & 0.95078 & 71 & 1.00 & 2.39 & 1.16 \\
FEM (RDFs; 3rd degree polynomials) & 0.95184 & 33 & 1.93 & 1.52 & 0.39 \\
FEM (RDFs; 5th degree polynomials) & 0.95177 & 27 & 0.45 & 0.46 & 0.47 \\
Heterogeneous & 0.95149 & & & & \\
\hline \hline
\end{tabular}

when the discontinuity factors are not used, we can decrease the error with the inclusion of the ADFs in the FEM. Again there is a good agreement between the FEM and PARCS when both use ADFs. The when using RDFs and RXSs we observe that, for degree 3, results show lower error in the power, while the errors in the flux are still quite large. This is due to different signs for the fast and for the thermal flux approximation errors, being canceled when calculating the power by chance. Increasing the polynomial degree for the RDFs solve the problem, giving a very good approximation (assembly errors smaller than $0.5 \%$ ) for both fluxes and for the power. 
In order to show the homogenization process, Figures 9 and 10 show the heterogeneous and homogenized solutions for the fast and the thermal neutron flux for the CISE reactor. It can be seen, that the homogenized calculation also obtains discontinuous neutron fluxes in the 2D CISE benchmark problem.

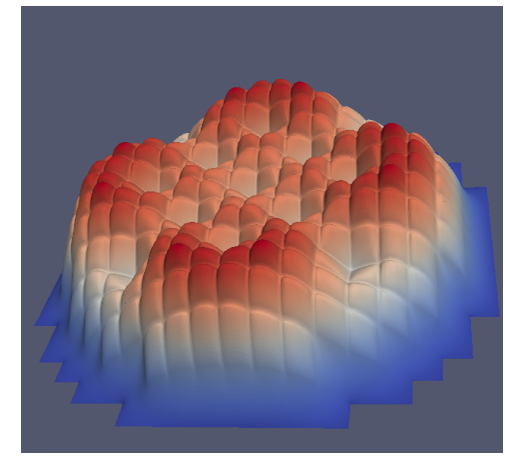

(a) Heterogeneous solution

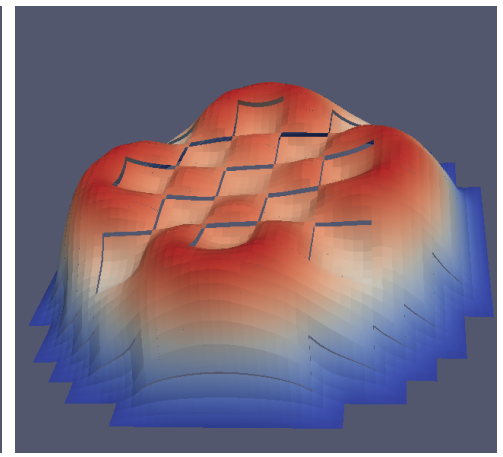

(b) Homogenized solution with ADFs

Figure 9: Fast flux for the CISE core.

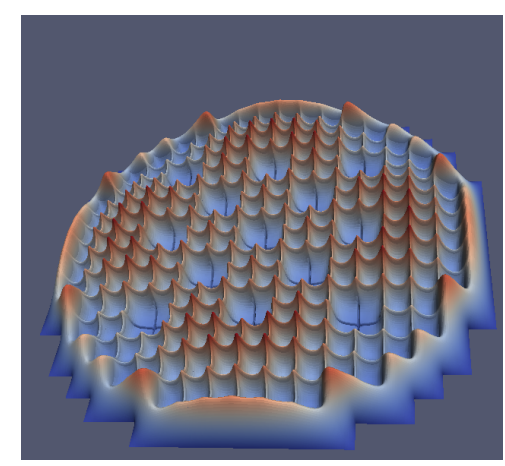

(a) Heterogeneous solution

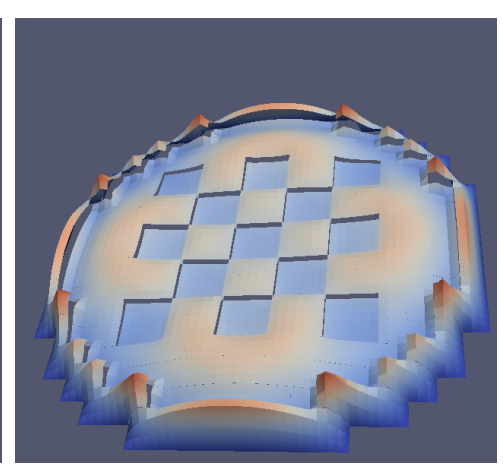

(b) Homogenized solution with ADFs

Figure 10: Thermal flux for the CISE core.

\section{Conclusions}

A detailed description of a nuclear power reactor core taking into account the detailed composition of the different materials composing the fuel assemblies is a very expensive task from the computational point of view, even when a low order approximation of the neutron transport equation such as the neutron diffusion equation is used. Thus, a homogenized version of the core is generally used for whole core reactor calculations. The use of assembly flux discontinuity factors has been proposed to improve the homogenization process, searching for discontinuous solutions for the neutron flux. The assembly discontinuity factors conditions have been successfully implemented in nodal methods, since the unknowns of these methods are the average neutron fluxes and currents in the nodes. To increase the accuracy obtained with the nodal calculations, high order finite element methods have been proposed to discretion the neutron diffusion equation. In this work, we propose a discontinuous Galerkin method that introduces the assembly discontinuity factors condition in a weak form, by means of an interior penalty method, obtaining discontinuous solutions for the neutronic flux. The performance of the method have been tested with 1D and 2D benchmark problems, using both reference homogenized cross sections together with reference discontinuity factors, and assembly cross sections together with assembly discontinuity factors obtained with the standard homogenization method. It is 
shown that the finite element method proposed has a very good performance, being the most part of the errors found due to the homogenization process.

It has also been observed that the FEM could need to increase the polynomial degree to more than 3 for some problems in order to achieve enough accuracy. It could be automatized by means of an local mesh refinement based on error estimator, so the method will automatically reduce the spatial truncation error, being the homogenization error then the dominant one. This should be considered future works, together with different improvements of the homogenization error.

\section{Acknowledgements}

This work has been partially supported by the spanish Ministerio de Ciencia e Innovación under project ENE 201122823 and the Generalitat Valenciana under the project PROMETEO II/2014/008.

\section{References}

Arnold, D. N., Brezzi, F., Cockburn, B., Marini, D., 2000. Discontinuous Galerkin methods for elliptic problems. In: Cockburn, B., Karniadakis, G., Shu, C. (Eds.), Discontinuous Galerkin methods (Newport, RI, 1999). Vol. 11 of Lect. Notes Comput. Sci. Eng. Springer, Berlin, pp. 89-101.

Arnold, D. N., Brezzi, F., Cockburn, B., Marini, L. D., 2002. Unified analysis of discontinuous Galerkin methods for elliptic problems. SIAM journal on numerical analysis 39 (5), 1749-1779.

Bangerth, W., Hartmann, R., Kanschat, G., 2007. deal.II — a General Purpose Object Oriented Finite Element Library. ACM Trans. Math. Softw. 33 (4), 24.

Brezzi, F., Cockburn, B., Marini, L. D., Süli, E., 2006. Stabilization mechanisms in discontinuous Galerkin finite element methods. Computer Methods in Applied Mechanics and Engineering 195 (25), 3293-3310.

Chiang, R. T., 1982. Lattice homogenization theory for coarse mesh diffusion analysis. Proc. Topl. Mtg. Advances in Reactor Physics and Thermal Hydraulics, 391-405.

Davidson, G. G., Evans, T. M., Jarrell, J. J., Slaybaugh, R. N., 2011. Massively Parallel, Three-Dimensional Transport Solutions for the kEigenvalue Problem. In: Proc. International Conference on Mathematics and Computational Methods Applied to Nuclear Science \& Engineering (M\& C 2011), Brazil.

Epshteyn, Y., Rivière, B., 2007. Estimation of penalty parameters for symmetric interior penalty Galerkin methods. Journal of Computational and Applied Mathematics 206 (2), 843-872.

González-Pintor, S., Ginestar, D., Verdú, G., 2009. High Order Finite Element Method for the Lambda modes problem on hexagonal geometry. Annals of Nuclear Energy 36 (9), 1450-1462.

Hébert, A., 1985. Application of the Hermite method for finite element reactor calculations. Nuclear Science and Engineering 91 (34-58), 45.

Hébert, A., 2008. A Raviart-Thomas-Schneider solution of the diffusion equation in hexagonal geometry. Annals of Nuclear Energy 35 (3), 363-376.

Joo, H. G., Barber, D., Jiang, G., Downar, T. J., 1998. PARCS: A multi-dimensional two-group reactor kinetics code based on the nonlinear analytic nodal method. Purdue University.

Lesaint, P., Raviart, P. A., 1974. On a Finite Element Method for Solving the Neutron Transport Equation. Univ. Paris VI, Labo. Analyse Numérique. Palmtag, S. P., 1997. Advanced nodal methods for mox fuel analysis. Ph.D. thesis, Massachusetts Institute of Technology.

Park, K. W., Cho, N. Z., 2001. The p-version of the finite element method for the solution of two-dimensional neutron diffusion equations. Journal of Nuclear Science and technology 38 (4), 242-253.

Rahnema, F., Nichita, E. M., 1997. Leakage corrected spatial (assembly) homogenization technique. Annals of Nuclear Energy 24 (6), $477-488$.

Reed, W. H., Hill, T. R., 1973. Triangular mesh methods for the neutron transport equation. Los Alamos Report LA-UR-73-479.

Rivière, B., 2008. Discontinuous Galerkin methods for solving elliptic and parabolic equations: theory and implementation. Society for Industrial and Applied Mathematics.

Sanchez, R., 2009. Assembly homogenization techniques for core calculations. Progress in Nuclear Energy 51 (1), 14-31.

Sanchez, R., Dante, G., Zmijarevic, I., 2013. Diffusion Piecewise Homogenization via Flux Discontinuity Ratios. Nuclear Engineering and Technology 45 (6), 707-720.

Shahbazi, K., 2005. An explicit expression for the penalty parameter of the interior penalty method. Journal of Computational Physics 205 (2), 401-407.

Smith, K., 1986. Assembly homogenization techniques for light water reactor analysis. Progress in Nuclear Energy 17 (3), 303 - 335.

Stacey, W. M., 2007. Nuclear reactor physics. John Wiley \& Sons.

Sun, S., Wheeler, M. F., 2005. Symmetric and nonsymmetric discontinuous galerkin methods for reactive transport in porous media. SIAM Journal on Numerical Analysis 43 (1), 195-219.

Vidal-Ferrandiz, A., Fayez, R., Ginestar, D., Verdú, G., 2014. Solution of the Lambda modes problem of a nuclear power reactor using an h-p finite element method. Annals of Nuclear Energy 72 (0), 338-349.

Wagner, M. R., Koebke, K., 1983. Progress in nodal reactor analysis. Atomkernenergie Kerntechnik. ANS topical meeting on advances in reactor computations; Salt Lake City, UT (USA) 43 (2), 117-126. 
Wang, Y., Bangerth, W., Ragusa, J., 2009. Three-dimensional h-adaptivity for the multigroup neutron diffusion equations. Progress in Nuclear Energy $51(3), 543-555$ 